\title{
Applications of dissipative and anisotropic hydrodynamics in description of early stages of relativistic heavy-ion collisions
}

\author{
Wojciech Florkowski ${ }^{1,2, a}$ \\ ${ }^{1}$ Institute of Physics, Jan Kochanowski University, PL-25406 Kielce, Poland \\ ${ }^{2}$ The H. Niewodniczański Institute of Nuclear Physics, Polish Academy of Sciences, \\ PL-31342 Kraków, Poland
}

\begin{abstract}
Kinetic and hydrodynamic models describing early stages of relativistic heavy-ion collisions are discussed. We emphasise the role of the shear-bulk coupling for the correct determination of the time dependence of the bulk viscous pressure.
\end{abstract}

\section{Introduction}

Relativistic hydrodynamics plays an important role in modeling of relativistic heavy-ion collisions [1-13]. Initially, approaches based on the perfect fluid hydrodynamics were used. Nowadays, viscous codes are applied, since i) this allows for better description of the data and ii) there are general arguments that the fluid viscosity cannot be zero - this follows from the quantum mechanical considerations [14] as well as from the AdS/CFT correspondence [15].

In this note we analyse simple models describing the early stages of relativistic heavy-ion collisions and point out difficulties one may encounter in the application of dissipative hydrodynamics [16]. We connect these problems with an incomplete character of various computational schemes which are used to derive the viscous hydrodynamic equations. Our critical examination of the hydrodynamic approaches is based on the comparisons of the hydrodynamic results with the predictions of the underlying kinetic theory [17-19].

In Refs. $[17,18]$ we studied the effects connected with the shear viscosity and showed that recent formulations of second-order viscous hydrodynamics [20] agree better with the exact solutions of the kinetic equation than the standard Israel-Stewart approach $[1,2]$. In this work we concentrate in more detail on the effects connected with the bulk viscosity [21]. Recently, it has been found that the finite bulk viscosity coefficient leads to a better description of the flow harmonics in ultracentral collisions [22]. On the theoretical side, it has been shown that the correct description of the bulk viscous pressure demands the correct treatment of the bulk-viscous coupling [23, 24].

The recent methods used to improve the efficacy of the hydrodynamic approaches include: complete second-order treatments [20], third-order treatments [25, 26], and anisotropic hydrodynamics [27-33]. We argue that the improved description of dissipative processes can be achieved if one uses either the complete second-order approaches or anisotropic hydrodynamics (aHydro).

\footnotetext{
ae-mail: Wojciech.Florkowski@ifj.edu.pl
} 


\section{Dissipative and anisotropic hydrodynamics}

A standard hydrodynamic approach is based on the gradient expansion of the underlying phase-space distribution function around the local equilibrium state described by the Boltzmann (Bose-Einstein or Fermi-Dirac) distribution function. The corrections to local equilibrium give rise to various dissipative currents. At the early stages of heavy-ion collisions, such currents are quite large, since there exist large gradients present in the system (mainly due to the rapid longitudinal expansion). Even if the values of the kinetic coefficients are small, the corrections may become large as they are products of the kinetic coefficients and the gradients. This suggest using a complete second-order treatment of dissipative hydrodynamics which, in particular, includes the shear-bulk couplings.

The problems of second-order viscous hydrodynamics [16] triggered the development of reorganizations of viscous hydrodynamics in which large momentum-space anisotropies are built into the leading order of the hydrodynamic expansion [27-33]. The newly constructed framework is referred to as anisotropic hydrodynamics. It is important to stress that aHydro implicitly includes transport phenomena and their couplings.

\section{Kinetic equation for boost-invariant and transversally homogenous systems}

We have in mind early dynamics of the central rapidity region, hence we assume that the system is boost-invariant and azimuthally symmetric. In this case, our considerations may be based on the simple form of the kinetic equation

$$
\frac{\partial f}{\partial \tau}=\frac{f^{\mathrm{eq}}-f}{\tau_{\mathrm{eq}}}
$$

where $f(x, p)$ is the phase-space distribution function, $\tau=\sqrt{t^{2}-z^{2}}$ is the proper time, and $\tau_{\mathrm{eq}}$ is the relaxation time. The requirement of boost invariance implies that $f(x, p)$ may depend only on the three variables: $\tau, w$ and $p_{T}$. The boost-invariant variable $w$ is defined by the expression $w=t p_{L}-z E$, where $p_{L}\left(p_{T}\right)$ is the longitudinal (transverse) momentum and $E$ is the energy. The equilibrium background distribution function $f^{\text {eq }}$ may be written as

$$
f^{\mathrm{eq}}\left(\tau, w, p_{T}\right)=\frac{2}{(2 \pi)^{3}} \exp \left[-\frac{\sqrt{w^{2}+p_{T}^{2} \tau^{2}}}{T(\tau) \tau}\right],
$$

where $T$ is an effective temperature.

The first moment of the kinetic equation defines the divergence of the energy-momentum tensor that should be conserved.

$$
T^{\mu v}(\tau)=g_{0} \int d P p^{\mu} p^{v} f\left(\tau, w, p_{T}\right), \quad \partial_{\mu} T^{\mu v}=0 .
$$

Using the symmetry properties of the distribution function, we rewrite (3) in the form [27, 32]

$$
T^{\mu v}=\left(\mathcal{E}+\mathcal{P}_{T}\right) u^{\mu} u^{v}-\mathcal{P}_{T} g^{\mu v}+\left(\mathcal{P}_{L}-\mathcal{P}_{T}\right) z^{\mu} z^{v},
$$

where $u^{\mu}=(t, 0,0, z) / \tau$ and $z^{\mu}=(z, 0,0, t) / \tau$. The energy density and the two (longitudinal and transverse) pressures are defined as the integrals over the distribution function multiplied by the appropriate combinations of the momentum. The parameter $g_{0}$ in Eq. (3) is the degeneracy factor connected with internal degrees of freedom different than spin. 

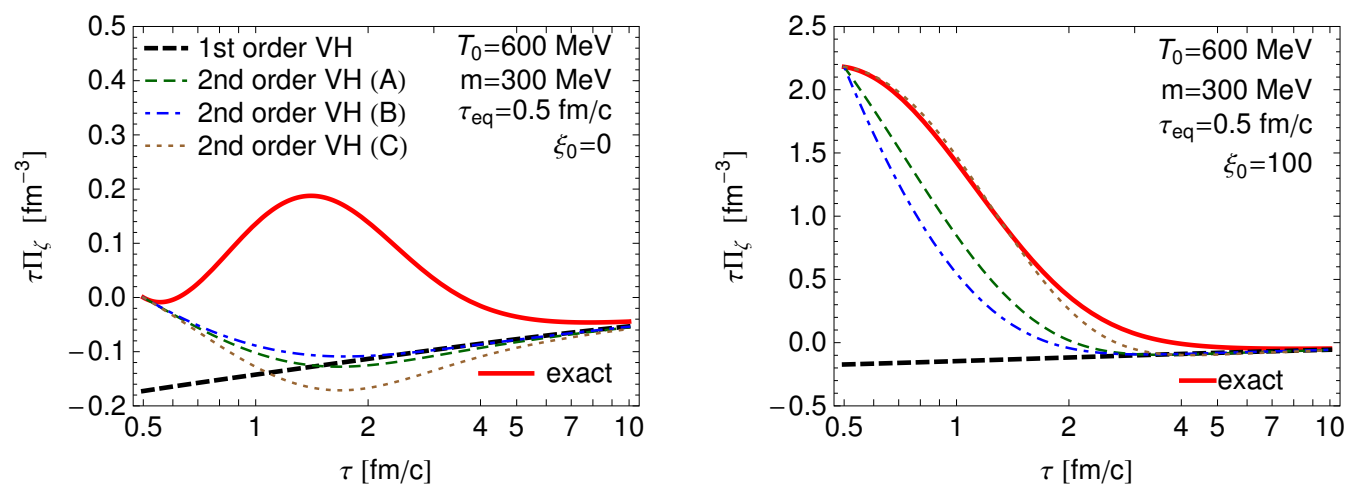

Figure 1. Time dependence of the bulk viscous pressure (multiplied by the proper time) obtained from the kinetic theory and various hydrodynamic equations.

The initial condition at the time $\tau=\tau_{0}$ used to solve numerically Eq. (1) corresponds to the Romatschke-Strickland (RS) form of the distribution function [34]

$$
f_{0}\left(w, p_{T}\right)=\frac{1}{4 \pi^{3}} \exp \left[-\frac{\sqrt{\left(1+\xi_{0}\right) w^{2}+p_{T}^{2} \tau_{0}^{2}}}{\Lambda_{0} \tau_{0}}\right] \text {. }
$$

This form reduces to an isotropic Boltzmann distribution if the anisotropy parameter $\xi_{0}=\xi\left(\tau_{0}\right)$ vanishes. In this case, the transverse momentum scale $\Lambda_{0}=\Lambda\left(\tau_{0}\right)$ is equal to the system's initial temperature $T_{0}$. The methods of solving Eq. (1) has been expained in more detail in [17-19].

If the solution of the kinetic equation is found, one can calculate the bulk viscous pressure from the equation

$$
\Pi_{\zeta}^{k}(\tau)=\frac{1}{3}\left[\mathcal{P}_{\|}(\tau)+2 \mathcal{P}_{\perp}(\tau)-3 \mathcal{P}_{\mathrm{eq}}(\tau)\right]
$$

where the equilibrium pressure $\mathcal{P}_{\text {eq }}$ is connected with the energy density $\mathcal{E}$ by the equation of state (for the massive gas). In Fig. 1 the red solid curves show the time dependence of the bulk viscous pressure calculated from the kinetic equation for the two different initial conditions characterised by the momentum anisotropy parameter $\xi_{0}$. In the two cases the initial temperature of the system is $T_{0}=600 \mathrm{MeV}$, the effective particle mass is $m=300 \mathrm{MeV}$, and the equilibration time $\tau_{\mathrm{eq}}=0.5 \mathrm{fm}$.

\section{Bulk viscous pressure in dissipative and anisotropic hydrodynamics}

In the most popular formulations of dissipative hydrodynamics, the bulk viscous pressure evolution is determined by one of the following three equations:

$$
\begin{aligned}
\tau_{\Pi} \dot{\Pi}_{\zeta}+\Pi_{\zeta} & =-\frac{\zeta}{\tau}-\frac{1}{2} \tau_{\Pi} \Pi_{\zeta}\left[\frac{1}{\tau}-\left(\frac{\dot{\zeta}}{\zeta}+\frac{\dot{T}}{T}\right)\right], \\
\tau_{\Pi} \dot{\Pi}_{\zeta}+\Pi_{\zeta} & =-\frac{\zeta}{\tau}-\frac{4}{3} \tau_{\Pi} \Pi_{\zeta} \frac{1}{\tau},
\end{aligned}
$$




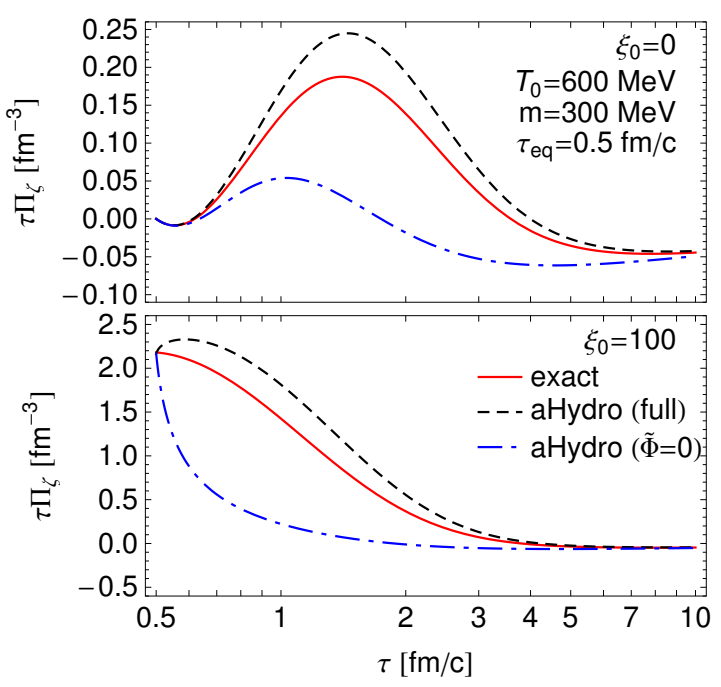

Figure 2. Time dependence of the bulk viscous pressure for two different initial anisotropy parameters: $\xi_{0}=0$ (upper panel) and $\xi_{0}=100$ (lower panel). The solid lines show the exact result. The dashed-dotted lines represent the predictions of aHydro formulated in Ref. [36], while the dashed lines represent the predictions of Ref. [37].

$$
\tau_{\Pi} \dot{\Pi}_{\zeta}+\Pi_{\zeta}=-\frac{\zeta}{\tau}
$$

We have solved Eqs. (7)-(9) with the initial conditions matching those used in the kinetic-theory approach. The results are shown in Fig. 1. The cases (A), (B), and (C) correspond to Eqs. (7), (8), and (9), respectively. The thick dashed line describes the first order hydrodynamic result where the bulk pressure is directly connected with the bulk viscosity through the formula $\Pi_{\zeta}=-\zeta / \tau$. The striking result of our comparisons is that none of the hydrodynamic equations (7)-(9) can properly reproduce the numerical result.

In the aHydro approach defined in Ref. $[35,36]$, one assumes that the leading-order phase-space distribution function has always the RS form with the parameters $\xi$ and $\Lambda$ depending on the proper time $\tau$. The dynamic equations are constructed from the first and second moments of the Boltzmann equation (1). In a more recent formulation of aHydro [37], the leading-order phase-space distribution function depends additionally on a parameter $\tilde{\Phi}$, and the dynamic equations follow from the zeroth, first and second moments of the kinetic equation (1) ${ }^{1}$. The results obtained with the two versions of aHydro are shown in Fig. 2, where we compare them with the results of the kinetic theory. One observes that the inclusion of an additional parameter in the ansatz for the distribution function helps to get a reasonable agreement with the kinetic theory. We also show that aHydro describes the evolution of the bulk pressure much better than the dissipative hydrodynamics based on Eqs. (7), (8), or (9).

Discrepancies between the results of the kinetic theory and dissipative hydrodynamics may be connected with the absence of the shear-bulk coupling in Eqs. (7), (8), and (9). In Fig. 3 we show the results for the bulk pressure with the shear-bulk coupling included [23] (dashed-dotted lines) and

\footnotetext{
${ }^{1}$ There exist also other formulations of aHydro, where some part of anisotropy is treated perturbatively by adding corrections to the leading-order term, see Ref. [38]
} 

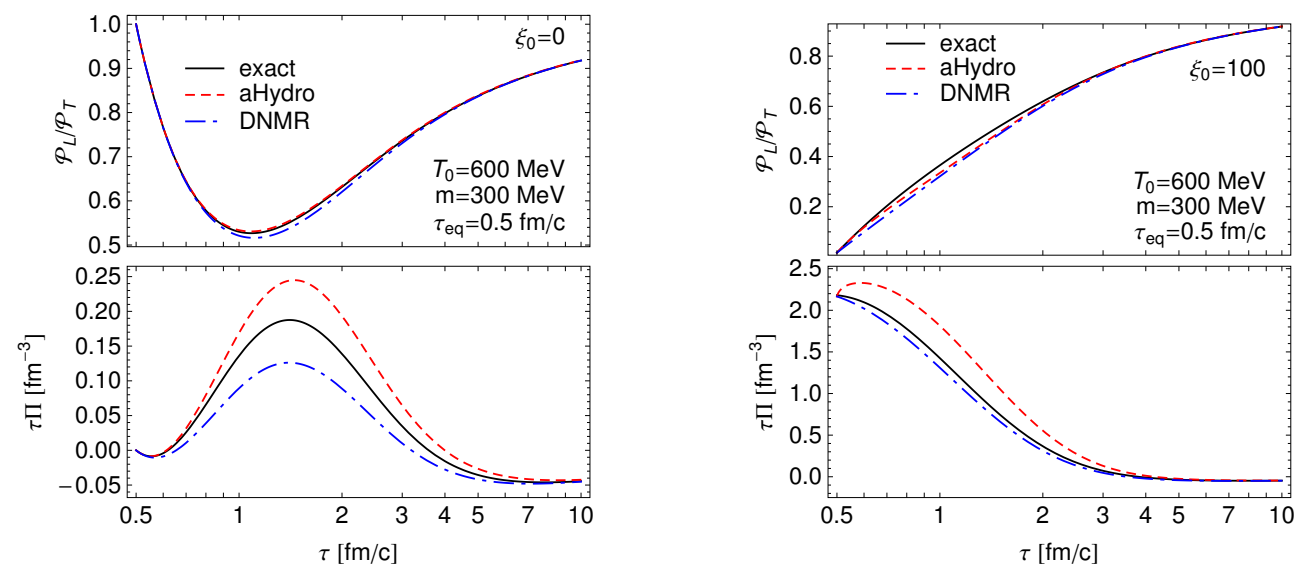

Figure 3. Time dependence of the ratio of the longitudinal and transverse pressure (upper panels) and of the bulk viscous pressure (lower panels). The left panels describe the cases where the initial anisotropy parameter vanishes, the right panels describe the cases where $\xi_{0}=100$. The kinetic theory results are shown as the solid lines, the complete second order hydrodynamic calculations including the shear-bulk coupling are represented by the dashed-dotted lines, and the aHydro results are shown as the dashed lines.

make comparisons with the kinetic theory results (solid lines ) and aHydro results [37] (dashed lines). The inclusion of the shear-bulk coupling improves the agreement between dissipative hydrodynamics and the exact kinetic theory solution. Similar conclusions have been also reached in Ref. [24].

\section{Conclusions}

Detailed comparisons between the exact results of the kinetic theory and the predictions of hydrodynamic models allow us to select the right structure of the hydrodynamic equations and the correct form of the kinetic coefficients. In this way we select the appropriate structure of the hydrodynamic equations that may be used to model relativistic heavy-ion collisions. We emphasise that the shear-bulk coupling is crucial for the correct determination of the time dependence of the bulk viscous pressure.

In this note we have presented the analysis of one-dimensional systems. Very recently, the exact solutions of the two-dimensional systems have become also available [39, 40] (for the systems which are boost-invariant and azimuthally symmetric) but they are restricted to conformal systems. Therefore, they are suitable for studies of the effects connected with shear viscosity.

Acknowledgments: I thank Gabriel Denicol, Ewa Maksymiuk, Radoslaw Ryblewski, Michael Strickland, and Leonardo Tinti for fruitful collaborations which have led us to the results presented in this paper. This work was supported in part by the Polish National Science Center with decision No. DEC-2012/06/A/ST2/00390.

\section{References}

[1] W. Israel and J. M. Stewart, Annals Phys. 118, 341 (1979). 
[2] A. Muronga, Phys. Rev. C 69, 034903 (2004).

[3] R. Baier, P. Romatschke and U. A. Wiedemann, Phys. Rev. C 73, 064903 (2006)

[4] P. Romatschke and U. Romatschke, Phys. Rev. Lett. 99, 172301 (2007).

[5] K. Dusling and D. Teaney, Phys. Rev. C 77, 034905 (2008).

[6] M. Luzum and P. Romatschke, Phys. Rev. C 78, 034915 (2008).

[7] H. Song and U. W. Heinz, J. Phys. G 36, 064033 (2009).

[8] G. S. Denicol, T. Kodama and T. Koide, J. Phys. G 37, 094040 (2010).

[9] B. Schenke, S. Jeon and C. Gale, Phys. Lett. B 702, 59 (2011).

[10] C. Shen, U. Heinz, P. Huovinen and H. Song, Phys. Rev. C 84, 044903 (2011).

[11] P. Bozek, Phys. Lett. B 699, 283 (2011).

[12] H. Niemi, G. S. Denicol, P. Huovinen, E. Molnar and D. H. Rischke, Phys. Rev. Lett. 106, 212302 (2011).

[13] P. Bozek and I. Wyskiel-Piekarska, Phys. Rev. C 85, 064915 (2012).

[14] P. Danielewicz and M. Gyulassy, Phys. Rev. D 31, 53 (1985).

[15] P. Kovtun, D. T. Son and A. O. Starinets, Phys. Rev. Lett. 94, 111601 (2005).

[16] M. Martinez and M. Strickland, Phys. Rev. C 79, 044903 (2009).

[17] W. Florkowski, R. Ryblewski and M. Strickland, Nucl. Phys. A 916, 249 (2013).

[18] W. Florkowski, R. Ryblewski and M. Strickland, Phys. Rev. C 88, 024903 (2013).

[19] W. Florkowski, E. Maksymiuk, R. Ryblewski and M. Strickland, Phys. Rev. C 89, 054908 (2014).

[20] G. S. Denicol, H. Niemi, E. Molnar and D. H. Rischke, Phys. Rev. D 85, 114047 (2012).

[21] G. S. Denicol, S. Jeon and C. Gale, arXiv:1403.0962 [nucl-th].

[22] J. B. Rose, J. F. Paquet, G. S. Denicol, M. Luzum, B. Schenke, S. Jeon and C. Gale, arXiv:1408.0024 [nucl-th].

[23] G. S. Denicol, W. Florkowski, R. Ryblewski and M. Strickland, arXiv:1407.4767 [hep-ph].

[24] A. Jaiswal, R. Ryblewski and M. Strickland, arXiv:1407.7231 [hep-ph].

[25] A. El, Z. Xu and C. Greiner, Phys. Rev. C 81, 041901 (2010).

[26] A. Jaiswal, Phys. Rev. C 88, 021903 (2013).

[27] W. Florkowski and R. Ryblewski, Phys. Rev. C 83, 034907 (2011).

[28] M. Martinez and M. Strickland, Nucl. Phys. A 848, 183 (2010).

[29] R. Ryblewski and W. Florkowski, J. Phys. G 38, 015104 (2011).

[30] M. Martinez and M. Strickland, Nucl. Phys. A 856, 68 (2011).

[31] R. Ryblewski and W. Florkowski, Eur. Phys. J. C 71, 1761 (2011).

[32] M. Martinez, R. Ryblewski and M. Strickland, Phys. Rev. C 85, 064913 (2012).

[33] R. Ryblewski and W. Florkowski, Phys. Rev. C 85, 064901 (2012).

[34] P. Romatschke and M. Strickland, Phys. Rev. D 68, 036004 (2003).

[35] W. Florkowski and R. Ryblewski, Phys. Rev. C 85, 044902 (2012).

[36] W. Florkowski, R. Ryblewski, M. Strickland and L. Tinti, Phys. Rev. C 89, 054909 (2014).

[37] M. Nopoush, R. Ryblewski and M. Strickland, Phys. Rev. C 90, 014908 (2014).

[38] D. Bazow, U. W. Heinz and M. Strickland, arXiv:1311.6720 [nucl-th].

[39] G. S. Denicol, U. W. Heinz, M. Martinez, J. Noronha and M. Strickland, arXiv:1408.5646 [hepph].

[40] G. S. Denicol, U. W. Heinz, M. Martinez, J. Noronha and M. Strickland, arXiv:1408.7048 [hepph]. 